\title{
Hygromorphic response dynamics of oak: towards accelerated material characterization
}

\author{
T. Arends $\cdot$ L. Pel $\cdot$ H. P. Huinink
}

Received: 28 January 2017 / Accepted: 27 April 2017/Published online: 15 May 2017

(C) The Author(s) 2017. This article is an open access publication

\begin{abstract}
When a wood board is exposed to a change in relative humidity on only one of its surfaces, e.g. in case of flooring or a panel painting, the resulting asymmetric moisture content profile induces differential expansion over the thickness. Consequently a bending moment causes the board to curve. A theory is presented to describe the bending of a wood board due to a step change in relative humidity. The board is assumed to be homogeneous, isotropic, and linearly elastic. Moisture transport is presumed to obey the diffusion equation with constant coefficients, such that moisture transport can be directly related to the bending of the board. It is shown that the transient deflective behavior provides the diffusion coefficient and the final length change yields the linear hygroscopic expansion coefficient. Derived diffusion coefficients are in good agreement with values in literature. Furthermore, a scaling law for the deflection of the board is proposed, which is seen to be followed qualitatively but not quantitatively by experiments. Finally, by assuming the deflection of the board to be the response of a linear system, the deflective
\end{abstract}

Electronic supplementary material The online version of this article (doi:10.1617/s11527-017-1043-5) contains supplementary material, which is available to authorized users.

T. Arends $\cdot$ L. Pel $(\bowtie) \cdot$ H. P. Huinink

Department of Applied Physics, Eindhoven University

of Technology, P.O. Box 513, 5600 MB Eindhoven,

The Netherlands

e-mail: 1.pel@tue.nl frequency response of the board can be predicted from its step response. The results allow upscaling of deflection and expansion, such that behavior of thick boards can be determined from an experiment using a thin board.

Keywords Wood - Moisture transport . Hygromechanical behavior $\cdot$ Non-destructive evaluation

\section{Introduction}

The shape change due to moisture sorption is a familiar phenomenon in natural systems. A wellknown example of such a hygromorphic effect is the opening and closing of pine cones at different environmental conditions [1]. Due to the bilayered structure of the individual scales, a change in the relative humidity of the ambient air causes differential expansion of the two layers. As these two layers are firmly attached to each other this will result in a bending moment, thereby opening the cone. Another well-known example of a hygromorphic material is wood. Upon a change in the relative humidity of the ambient air, wood will exchange moisture with the ambient air [2], resulting in moisture transport in the material in order to reach an equilibrium. The resulting change in moisture content of the wood leads to expansion or shrinkage, as the fibers which constitute 
the wood are pushed apart by the entering moisture or approach when moisture is released. This property of wood has been used e.g. in mechanical hygrometers [3], or in barrels ensuring the sealing of the barrel. The anisotropic nature of wood, with differences in expansion in its three principal directions, can also induce out-of-plane deformation. The warping of wood boards has been applied in the construction of 19th century boathouses in Nordmøre, Norway [4]. The walls of these boathouses are cladded with pine boards, nailed towards their upper edges. The anisotropy in expansion causes the boards of the boathouse to bend upwards during dry conditions, allowing natural ventilation through the façade. During humid conditions, the boards bend downwards, ensuring weathertight sealing. More recently, a prototype façade has been developed which reacts to environmental changes by the warping of individual thin wood slices constituting the façade [5]. The effect may be amplified by adding a passive layer with different expansion to the active wood veneer, e.g. a polymer [6], thereby mimicking a pine cone or a bimetallic strip. Bending in wood can also be caused by differential expansion resulting from an asymmetric moisture content distribution. This phenomenon has been studied during kiln-drying [7-11], and in the case of a board simulating a painted panel [12].

The expansion and shrinkage of wood are, however, more commonly associated with stresses in the material [13]. When these stresses exceed the strength of the material, cracks can be formed. These cracks are aesthetically undesired when a wooden art object is concerned [14], or in the case of a wooden parquet floor [15]. Cracks can also have consequences in a structural component, which can ultimately give rise to structural failure. A wide variety of experimental methods has been used to study the hygromorphic behavior of wood, e.g. using displacement measurement gauges [16], a linear variable differential transformer mounted to a micrometer [17], phase contrast synchrotron X-ray tomography [18], digital image correlation [19, 20], a combination of nuclear magnetic resonance (NMR) and a fiber Bragg grating optical sensor [21], or a combination of NMR and $\mathrm{X}$-ray microtomography [22]. All these methods are either complex or require sophisticated post-processing to derive useful, generalizable knowledge about the relation between moisture transport and expansion. Alternatively one could relate moisture transport to hygromorphic macroscopic behavior by analyzing the transient morphological or mechanical behavior during non-equilibrium conditions. Scherer and co-workers [23-26] have derived transport properties of different porous media from a bending experiment. The method relies on the redistribution of liquid in the pores of a liquid-saturated porous beam when subjected to three-point bending. Its mechanical response at constant deflection is influenced by the liquid flow and thus provides information about the permeability of the material. Moisture-induced bending of a beam of clay-bearing stone exposed to water on one side was thoroughly analyzed by Scherer and Jimenez-Gonzalez [27]. Similarly, Holmes et al. [28] considered the bending of gel beams when wetted unilaterally by hexane.

The basic idea in this study, shown in Fig. 1, is analogous. Here we will consider a wood board with a thickness much smaller than its length and width, clamped at one end. The board has an impermeable layer on five surfaces, leaving one surface exposed to the atmosphere surrounding it. A semi one-dimensional moisture transport experiment is thus created. Here we will assume that the impermeable layer has no influence on the mechanical behavior of the board. The board itself is initially in equilibrium with the surrounding air, and therefore has a flat moisture content profile (Fig. 1a) when suddenly the relative humidity of the surrounding air is changed. The moisture content at the exposed surface consequently rises, and moisture is transported into the material resulting in a moisture content gradient. The consequent asymmetric moisture profile causes unequal expansion over the thickness; the expansion near the exposed surface is the highest and decreases towards the coated surface. These differences in expansion result in a constant moment, and as a consequence the board bends (Fig. 1b). Due to moisture transport the moisture content profile continuously changes and consequently the bending changes continuously over time. The deflection has a maximum in time when the moment induced by the differences in expansion is the largest. Progressing in time, the moisture starts to equilibrate throughout the sample and the moisture content becomes homogeneous again. In the final situation the board is straight again, but elongated due to the increase in moisture content (Fig. 1c). Since the process is governed by moisture transport, one can expect that the dynamics of the bending is dependent 


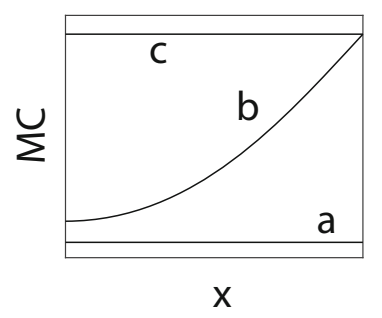

(a)

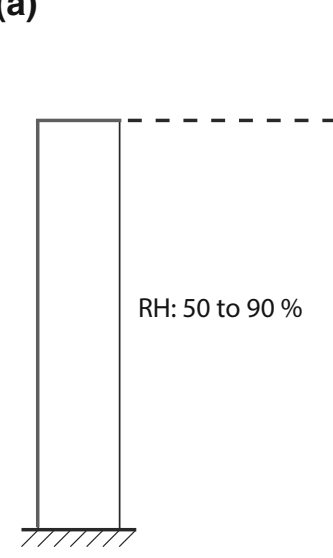

(b)

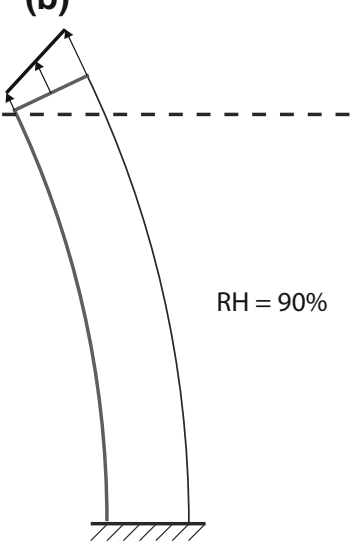

(c)

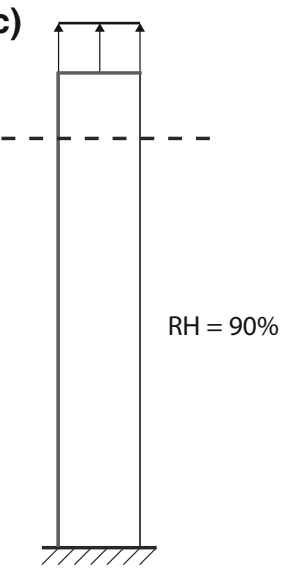

Fig. 1 The conceptual idea of the bending experiment used in this study. A board of hygroscopic material is exposed to a step change in the ambient relative humidity ( $\mathrm{RH})$ at one side. The board is sealed on all sides except the one exposed to the environment resulting in a one-dimensional moisture transport. a Initially the sample is in equilibrium and the moisture content

on both object geometry, in this case thickness, and material properties such as diffusivity and moisture expansion.

The aim of this study is the analysis of the dynamic moisture-induced bending of a wooden board. A theoretical model is introduced by coupling moisture transport to a linear elastic model for the moistureinduced bending. We analyze the bending in time after a sudden increase in relative humidity and couple it to internal moisture transport. We will first discuss the theory. Experimental verification is provided with boards with different thicknesses. The postulated step change in relative humidity is, however, not very realistic since changes of environmental conditions are continuous, generally manifested in daily or seasonally repeating cycles [29]. Nevertheless, the hygromorphic step response of the board can provide information on the frequency response characteristics. The behavior at very low frequencies, which is timeconsuming to determine experimentally, can thus be retrieved from a short experiment. The experimental study of moisture transport in wood during cycles is
(MC) is constant throughout the sample. b A sudden increase in the ambient relative humidity causes a moisture content gradient in the sample resulting in a bending moment due to differential expansion. $\mathbf{c}$ In the final situation the moisture has equilibrated throughout the sample and the board is straight again, but elongated due to a higher moisture content

often limited to block waves; examples investigating sinusoidal fluctuations are scarce $[16,17,30]$. We will discuss the theory to derive the frequency response, and the experimental verification with sinusoidal relative humidity fluctuations.

\section{Hygro-bending theory}

In this section, the bending of a clamped hygromorphic board as shown schematically in Fig. 2, sealed on five of its six sides, is described by introducing a socalled hygro-bending theory. First the moisture transport arising from a sudden change of the ambient relative humidity will be described analytically. A simple, linear elastic model is then proposed to express the consequent bending of the board.

\subsection{Moisture transport}

A step change in the moisture content at the open surface of the board from $c_{O}$ to $c_{d}$ will result in one- 
Fig. 2 Schematic representation of a board as used in the theory of the bending. The board has an initial length $L_{O}$ and thickness $d$, and is exposed on one surface to a change in moisture content from $c_{0}$ to $c_{d}$

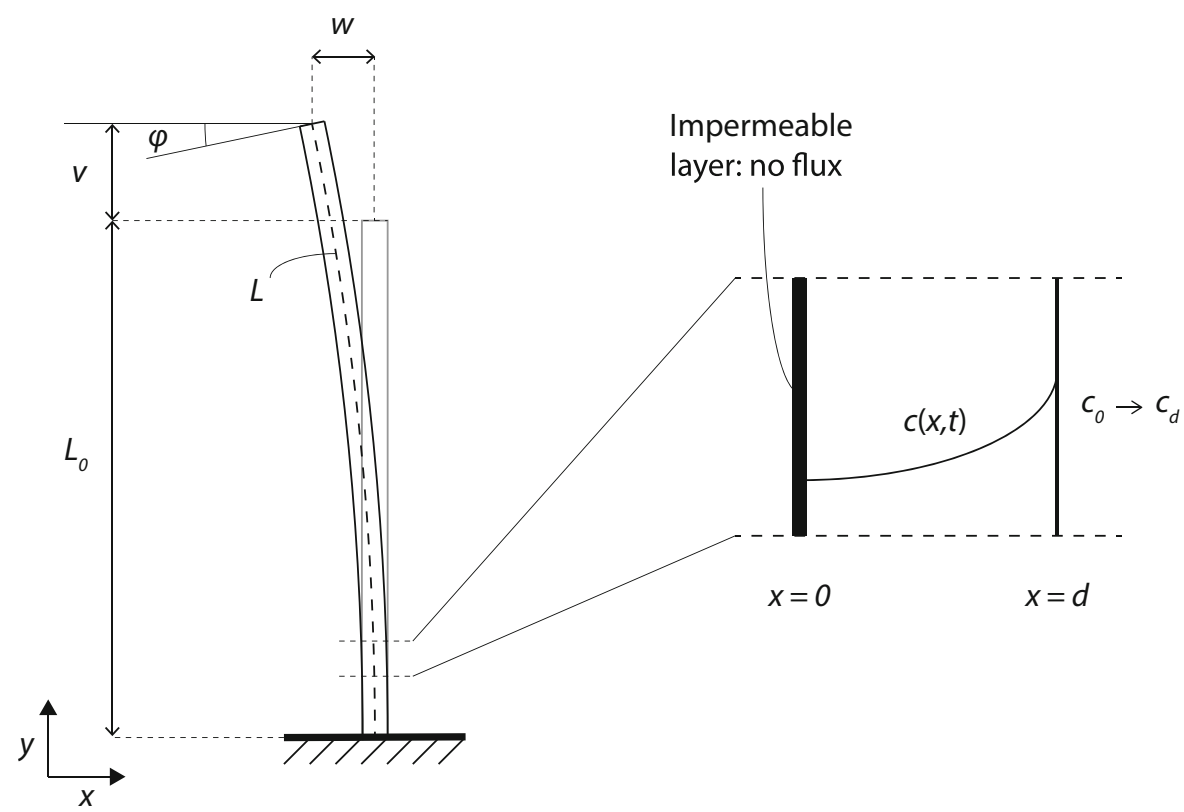

dimensional moisture transport. Here we will assume that moisture transport is characterized by a constant diffusion coefficient $D$ throughout the thickness $d$ of the board. The other surface is sealed and therefore has a no-flux boundary condition. Mathematically this can be formulated as:

$$
\left\{\begin{array}{l}
\partial_{t} c=D \partial_{x x} c \\
c(x, 0)=c_{0} \\
c(d, t)=c_{d} \\
\partial_{x} c(0, t)=0
\end{array}\right.
$$

where $c$ is the moisture content in $\mathrm{kg}$ water $/ \mathrm{kg}$ dry wood. An analytical solution can be obtained by separation of variables, see e.g. [31]. The result can be formulated in the form of the Fourier series

$$
\begin{aligned}
c(x, t)= & c_{d}-\frac{4}{\pi}\left(c_{d}-c_{0}\right) \sum_{n=0}^{\infty} \frac{(-1)^{n}}{2 n+1} e^{-\left(\frac{2 n+1}{2 d} \pi\right)^{2} D t} \\
& \cos \left(\frac{2 n+1}{2 d} \pi x\right) .
\end{aligned}
$$

A second solution can be formulated when the medium can be considered semi-infinite, i.e. when the penetration depth of the moisture $l$ is small compared to the thickness of the board $(l / d \ll 1)$. In this regime, the moisture concentration can be described as $c(x, t)=c_{0}+\left(c_{d}-c_{0}\right) \operatorname{erf} c\left(\frac{d-x}{2 \sqrt{D t}}\right)$,

with the penetration depth of the moisture

$l \propto \sqrt{D t}$.

\subsection{Transient bending}

\subsubsection{Coupling with moisture distribution}

A change in moisture content in a hygromorphic material results in expansion or shrinkage. In case of a uniform moisture distribution $c$ and no lateral contraction, the expansion of the board $\varepsilon$ is given by

$\varepsilon(t)=\frac{L(t)-L_{0}}{L_{0}}=\alpha\left(c(t)-c_{0}\right)$,

where $L$ is the length of the board at time $t, L_{O}$ the length of the board at $t_{0}, \alpha$ the linear hygroscopic expansion coefficient [3, 29], and $c_{0}$ the uniform moisture content at time $t_{0}$. After a step change in the moisture content at the open surface, the moisture content will not be uniform. As a result the timeevolution of the expansion of the board can no longer be described by Eq. (5). Furthermore, the board bends 
due to the uneven expansion across its thickness. In order to describe the bending of the board, the assumption is made that the material is linearly elastic with no lateral contraction (for a more in-depth discussion see the strictly analogous problem of thermal expansion in chapter 10 of Boley and Weiner [32]). By doing so, the expansion of the board at halfthickness $\varepsilon$ can be expressed as

$\varepsilon(t)=\frac{\alpha}{d} \int_{0}^{d}\left(c(x, t)-c_{0}\right) \mathrm{d} x$.

Note that for a constant moisture content, Eq. (6) simplifies to Eq. (5). The bending of the board can be characterized by the angle $\theta$ of its cross-section (see Fig. 2):

$\theta(t)=12 \frac{\alpha L}{d^{3}} \int_{0}^{d}\left(c(x, t)-c_{0}\right)\left(x-\frac{d}{2}\right) \mathrm{d} x$.

Here the integral represents the moment exerted on the board as a consequence of an unsymmetrical moisture content distribution. It can be shown that for the general case of a symmetric moisture content profile, i.e. $c(x, t)=c(d-x, t)$, the integral in Eq. (7) vanishes and the angle of the cross-section of the board equals zero. Hence in case of a two-sided exposure to a change in relative humidity there will be no deflection, only an expansion in the y-direction. In case of a onesided step change, the moisture profile will approach the constant value $c_{d}$ for $t \rightarrow \infty$, and consequently the angle $\theta$ approaches zero again.

The bending of the board causes deflection, which is maximal at the free end of the board. The $\mathrm{x}$ deflection at the free end at half-thickness is given by

$w(t)=\frac{L}{\varphi}(1-\cos \varphi)$,

and the corresponding $\mathrm{y}$-deflection can be expressed as

$v(t)=\frac{L}{\varphi} \sin \varphi-L_{0}$,

where $\varphi=\tan ^{-1} \theta$. Under the assumption that the deflection of the beam remains small, i.e. $\varphi \approx \theta$, a compact expression for the deflection can be derived by taking into account only the first two terms of the Taylor series expansion of Eq. (8) and assuming $L_{O} \approx L$ : $w(t)=6 \frac{\alpha L_{0}^{2}}{d^{3}} \int_{0}^{d}\left(c(x, t)-c_{0}\right)\left(x-\frac{d}{2}\right) \mathrm{d} x$.

\subsubsection{Explicit expressions for expansion and deflection}

We have now obtained analytical expressions to describe the expansion of the board at half-thickness and the deflection of its free end following a step change in the moisture content at one surface. We also introduced two expressions for the moisture content evolution. Three expressions for the expansion and the deflection can be constructed with the two descriptions of the moisture content evolution. First of all, a total solution for the expansion $\varepsilon_{t o t}$ and the deflection $w_{t o t}$ is obtained by substitution of Eq. (2) into the expressions for $\varepsilon$ and $w$ in Eqs. (6) and (10), which results in

$\varepsilon_{t o t}(t)=\alpha\left(c_{d}-c_{0}\right)\left(1-\frac{8}{\pi^{2}} \sum_{n=0}^{\infty} \frac{1}{(2 n+1)^{2}} e^{-\left(\frac{2 n+1}{2} \pi\right)^{2} \frac{D t}{d^{2}}}\right)$.

and

$w_{\text {tot }}(t)=24 \frac{\alpha L_{0}^{2}}{\pi^{2} d}\left(c_{d}-c_{0}\right) \sum_{n=0}^{\infty}\left(\frac{4(-1)^{n}}{\pi(2 n+1)^{3}}-\frac{1}{(2 n+1)^{2}}\right) e^{-\left(\frac{2 n+1}{2} \pi\right)^{2} \frac{D_{t}}{d^{2}}}$.

Note that the expansion and deflection in Eqs. (11) and (12) can be rewritten as

$$
\begin{array}{r}
\varepsilon(t)=\alpha\left(c_{d}-c_{0}\right) \varepsilon^{*}\left(t^{*}\right), \\
w(t)=\frac{\alpha L_{0}^{2}}{d}\left(c_{d}-c_{0}\right) w^{*}\left(t^{*}\right),
\end{array}
$$

where $\varepsilon^{*}$ and $w^{*}$ are the scaled expansion and deflection respectively, as a function of the dimensionless time $t^{*}=D t / d^{2}$. The scaled expansion and deflection for the total solution can accordingly be expressed as

$$
\begin{aligned}
& \varepsilon_{\mathrm{tot}}^{*}\left(t^{*}\right)=1-\frac{8}{\pi^{2}} \sum_{n=0}^{\infty} \frac{1}{(2 n+1)^{2}} e^{-\left(\frac{2 n+1}{2} \pi\right)^{2} t^{*}}, \\
& w_{\mathrm{tot}}^{*}\left(t^{*}\right)=\frac{24}{\pi^{2}} \sum_{n=0}^{\infty}\left(\frac{4(-1)^{n}}{\pi(2 n+1)^{3}}-\frac{1}{(2 n+1)^{2}}\right) e^{-\left(\frac{2 n+1}{2} \pi\right)^{2} t^{*}} .
\end{aligned}
$$


Note that, as in Eq. (2), the expressions contain an infinite sum of modes, which makes a concise analysis impossible. A second set of solutions for the expansion $\varepsilon_{M}$ and deflection $w_{M}$ can be obtained by taking into account only the first two modes:

$\varepsilon_{M}(t)=\alpha\left(c_{d}-c_{0}\right)\left(1-\frac{8}{\pi^{2}} e^{-\frac{\pi^{2} D t}{4 d^{2}}}-\frac{8}{9 \pi^{2}} e^{-\frac{9 \pi^{2} D t}{4 d^{2}}}\right)$,

and

$w_{M}(t)=24 \frac{\alpha L_{0}^{2}}{\pi^{2} d}\left(c_{d}-c_{0}\right)\left[\left(\frac{4}{\pi}-1\right) e^{-\frac{\pi^{2} D t}{4 d^{2}}}-\frac{1}{9}\left(1+\frac{4}{3 \pi}\right) e^{-\frac{9 \pi^{2} D t}{4 d^{2}}}\right]$

This results in a 'long-term' solution, since higher order modes are neglected and the terms with the smallest decay constants remain. At small times, the long-term solution deviates largely from the total solution. At large times, however, it will converge to the total solution, due to the dominance of the low modes. Similar to total solution, the long-term solution can also be rewritten according to Eq. (13), which yields for the scaled expansion and deflection:

$\varepsilon_{M}^{*}\left(t^{*}\right)=1-\frac{8}{\pi^{2}} e^{-\frac{\pi^{2}}{4} t^{*}}-\frac{8}{9 \pi^{2}} e^{-\frac{9 \pi^{2}}{4} t^{*}}$,

$w_{M}^{*}\left(t^{*}\right)=\frac{24}{\pi^{2}}\left[\left(\frac{4}{\pi}-1\right) e^{-\frac{\pi^{2}}{4} t^{*}}-\frac{1}{9}\left(\frac{4}{3 \pi}+1\right) e^{-\frac{9 \pi^{2}}{4} t^{*}}\right]$.

A third set of expressions for the expansion $\varepsilon_{S}$ and deflection $w_{S}$ can be obtained by substitution of the short-term solution, i.e. Eq. (3), in the expressions for $\varepsilon$ and $w$ to yield

$\varepsilon_{S}(t)=2 \frac{\alpha\left(c_{d}-c_{0}\right)}{d} \sqrt{\frac{D t}{\pi}}$,

and

$w_{S}(t)=6 \frac{\alpha L_{0}^{2}}{d^{3}}\left(c_{d}-c_{0}\right)\left(d \sqrt{\frac{D t}{\pi}}-D t\right)$.

The scaled expansion and deflection for the shortterm solution are then obtained as:

$\varepsilon_{S}^{*}\left(t^{*}\right)=\frac{2}{\sqrt{\pi}} \sqrt{t^{*}}$.
$w_{S}^{*}\left(t^{*}\right)=6\left(\sqrt{\frac{t^{*}}{\pi}}-t^{*}\right)$.

The scaled expansions, i.e. Eqs. (14), (18), and (22), are shown in Fig. 3a as a function of the square root of $t^{*}$. As can be seen, the short-term solution $\varepsilon_{S}^{*}$ is linear with a slope $2 / \sqrt{ } \pi$. This linear behavior is initially also seen in the total solution $\varepsilon_{t o t}^{*}$, up to a value for $\sqrt{ } t^{*}$ of $\sim 0.5$. The long-term solution deviates at low values of $t^{*}$, but converges to the total solution from a value for $\sqrt{ } t^{*}$ of $\sim 0.2$. The scaled deflections from Eqs. (15), (19), and (23) are plotted in Fig. 3b as a function of $t^{*}$. All three solutions rise up to a point of maximum deflection, after which the deflection decreases again. This is also apparent by comparing the long-term and the short-term solution for the scaled deflection. We observe that both solutions are composed of a rising component which is dominant at short times, and a negative component, which gains dominance at large times. In the long-term solution, these components are exponentials with different decay constants; in the short-term solution, these components are a square root and a linear term respectively. Nevertheless, the qualitative behavior is similar. Furthermore it is seen that the short-term solution follows the complete solution perfectly up to the point of maximum deflection, and starts to deviate considerably hereafter. The opposite is observed for the long-term solution, which deviates significantly for short times, but converges to the complete solution even before the point of maximum deflection. A hybrid solution can therefore be proposed, which consists of the short-term solution for times up to the maximum deflection, and the long-term solution for the deflection after the maximum.

\subsection{Derivation of parameters from transient data}

To find the time $t_{m}$ at which the maximum deflection $w_{m}$ occurs we can take the zero of the derivative with respect to time for the long-term and short-term solution, which, for both solutions, has the form

$t_{m}=\phi_{i} \frac{d^{2}}{D}$,

with 
Fig. 3 a The scaled expansion $\varepsilon^{*}$ and $\mathbf{b}$ the scaled deflection $w^{*}$ of the three different solutions (total, long-term, short-term, subscripts tot, $\mathrm{M}$, and $\mathrm{S}$ respectively) as a function of the square root of dimensionless time $t^{*}$

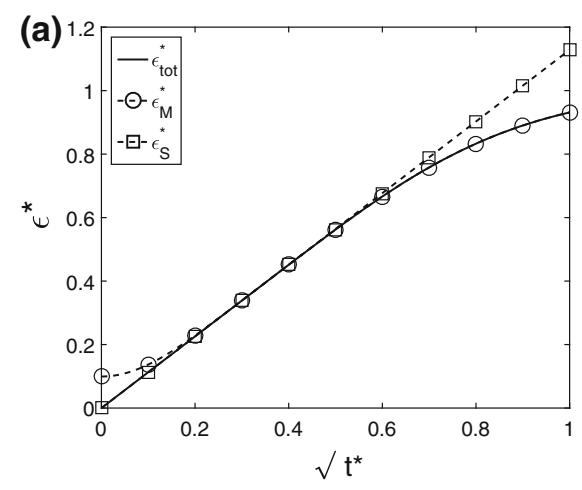

$\phi_{M}=\frac{1}{2 \pi^{2}} \ln \left(\frac{1}{3} \frac{3 \pi+4}{4-\pi}\right), \phi_{S}=\frac{1}{4 \pi}$.

Hence the time at which the board is deflected maximally is proportional to the typical diffusion time $d^{2} / D$. Inserting $t_{m}$ into Eqs. (17) or (21) yields the maximum deflection of the board

$w_{m}=\psi_{i} \frac{\alpha L_{0}^{2}}{d}\left(c_{d}-c_{0}\right)$,

with

$$
\begin{aligned}
\psi_{M}= & \frac{24}{\pi^{2}}\left[\left(\frac{4}{\pi}-1\right)\left(3 \frac{4-\pi}{3 \pi+4}\right)^{\frac{1}{8}}-\frac{1}{9}\left(\frac{4}{3 \pi}+1\right)\right. \\
& \left.\left(3 \frac{4-\pi}{3 \pi+4}\right)^{\frac{9}{8}}\right], \psi_{S}=\frac{3}{2 \pi} .
\end{aligned}
$$

The maximum deflection is thus proportional to the linear expansion coefficient, the length of the board squared, and the step in moisture content at the surface, and inversely proportional to the thickness of the board. Furthermore, the point of maximum deflection is similar for the long-term and short-term solution, with $\phi_{M} / \phi_{S}=1.05$ and $\psi_{M} / \psi_{S}=1.01$. Moreover, it appears that the maximum deflection is independent of the diffusion coefficient. The diffusion coefficient only plays a role in the time at which the maximum occurs. By performing an experiment with a board of thickness $d$, the diffusion coefficient can be derived from the results by rewriting Eq. (24) to arrive at

$$
D=\phi_{i} \frac{d^{2}}{t_{m}}
$$

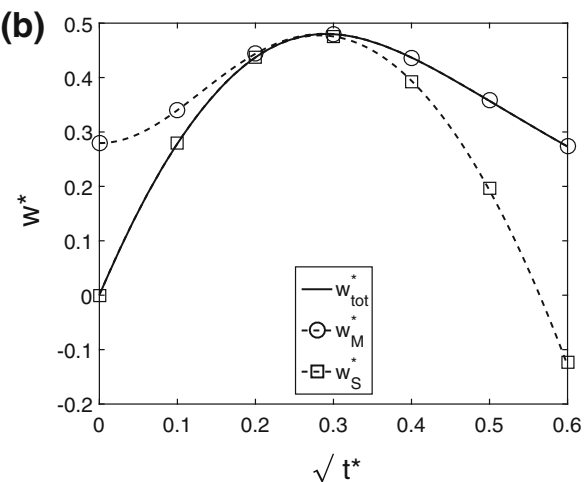

To determine the time of maximum deflection $t_{m}$, the $\mathrm{x}$-deflection is first fitted with the double exponential function

$x(t)=\beta_{0}\left(e^{-c_{1} t}-e^{-c_{2} t}\right)$,

where $\beta_{0}, c_{1}$, and $c_{2}$ are fitting parameters. Note that Eq. (29) has a similar form as Eq. (17). Equation (29) has a maximum at the time

$t_{m}=\frac{1}{c_{2}-c_{1}} \ln \left(\frac{c_{2}}{c_{1}}\right)$.

Substitution of Eq. (30) in Eq. (29) yields the maximum deflection. Moreover, the diffusion coefficient can be calculated by substitution of $t_{m}$ in Eq. (28). Analogous to the x-deflection, the y-deflection can be fitted by:

$y(t)=\beta_{3}\left(e^{-c_{3} t}-1\right)+\beta_{4}\left(e^{-c_{4} t}-1\right)$,

where $\beta_{3}, c_{3}, \beta_{4}$ and $c_{4}$ are fitting parameters. For $t \rightarrow \infty$ the $\mathrm{x}$-deflection approaches zero, and consequently the y-deflection approaches the length change of the board. Hence by analyzing the bending response of a board after a step change in the ambient relative humidity the diffusivity can be obtained. The linear hygroscopic expansion coefficient $\alpha$ can be derived from Eq. (16) as the asymptote for $t \rightarrow \infty$, in case the step in moisture content is known. Furthermore, Eq. (26) provides us a scaling law with which experimental results using boards of different thicknesses can be compared.

\subsection{From step response to frequency response}

To derive frequency response characteristics from a step response, the deflective behavior of the wood 
board is considered as the dynamic response of a linear system [33]. The frequency response for the $\mathrm{x}-$ deflection can be obtained by first determining the Laplace transform of Eq. (29) in terms of the complex variable $s$ :

$X(s)=\mathcal{L}(x(t))=\beta_{0}\left(\frac{1}{c_{1}+s}-\frac{1}{c_{2}+s}\right)$.

Equation (29) is the response of the linear system to a step change in the moisture content at the surface at $t=0$. Since the board is approximated by a linear system, only the difference in initial and final value of the moisture content is of interest, not the actual values. The step change $u(t)$ can therefore be written as

$u(t)=a$,

where $a$ is the step in moisture content. The Laplace transform of Eq. (33) is:

$U(s)=\frac{a}{s}$.

In a linear system, the Laplace transforms $X$ and $U$ of the output and input function respectively, are related by:

$X(s)=H(s) U(s)$,

where $H(s)$ is the transfer function of the system. Note that this relation holds for all input functions $u(t)$ for which a Laplace transform exists. As a consequence, if the transfer function of a system is known, the inverse Laplace transform of Eq. (35) will yield the transient response $x(t)$ of the system to an arbitrary input $u(t)$. Using Eqs. (32) and (34) in Eq. (35), the transfer function of the $\mathrm{x}$-deflection can be expressed as

$H(s)=\frac{s \beta_{0}\left(c_{2}-c_{1}\right)}{a\left(c_{1}+s\right)\left(c_{2}+s\right)}$.

If the transfer function of the system is known, its frequency response can be determined by considering the real and imaginary parts of $H(i \omega)$, with $\omega$ the angular frequency. $H(i \omega)$ is a vector in the complex plane; its magnitude is the amplitude of the steady-state output signal to a sine input signal with unit amplitude, and its angle with the positive real axis determines the phase shift of the output signal. Using these results, Bode plots can be constructed which show the amplitude on a logarithmic scale and phase on a linear scale as a function of frequency. A similar analysis can be performed to determine the frequency dependence of the y-deflection of the board. The transfer function of the y-deflection can be obtained using Eq. (34) and the Laplace transformation of Eq. (31) as

$G(s)=\frac{s\left(\beta_{3} c_{4}+\beta_{4} c_{3}+s\left(\beta_{3}+\beta_{4}\right)\right)}{a\left(c_{3}+s\right)\left(c_{4}+s\right)}$.

In a similar fashion, the frequency behavior of the $y$-deflection can be calculated by considering the real and imaginary part of $G(i \omega)$.

\section{Materials and methods}

Experiments were conducted on oak boards where the bending due to one-sided exposure to a change in relative humidity is assessed. First, the material of interest and the preparation of samples are described, after which the experimental procedure is explained with the description of the set-up.

\subsection{Material}

Oak boards with a length of $100 \mathrm{~mm}$, a width of $30 \mathrm{~mm}$, and different thicknesses $(2,4,6,8 \mathrm{~mm})$ were sawn from a large oak wood board. Because wood is an anisotropic material with properties dependent on the characteristic directions, attention is paid to the grain direction in the samples. All samples were prepared such that the grain direction is along the width of the board. Perpendicular to that, the tangential direction is in the length of the board, and the radial direction along the thickness. Transport hence occurs in the radial direction of the wood, whereas the expansion along the length of the board is the expansion in tangential direction. Since all experiments start at a relative humidity of $50 \%$, the boards were placed in a desiccator containing a saturated $\mathrm{Mg}\left(\mathrm{NO}_{3}\right)_{2} \cdot 6 \mathrm{H}_{2} \mathrm{O}$ solution, ensuring a relative humidity of $53 \%$. Prior to the experiment, Bison silicone kit $\subset$ was applied on five sides of the board, leaving one of the two largest surfaces open. The silicone kit was allowed to dry for at least 1 day.

\subsection{Experimental set-up}

The experimental set-up designed to measure the deflection of a wood board due to humidity fluctuations is shown in Fig. 4. One end of the wood board is clamped between two PVC strips. The other end of the 
sample is also clamped between two strips, on which a pointer is mounted. This setup is placed in a plastic container in which the relative humidity can be controlled by the help of a humidifier, which operates in the relative humidity range $0-95 \%$. A Dino-Lite $\subset$ digital microscope records images to measure the deflection. Using a Matlab optical recognition program the position of the pointer is recorded automatically.

In the humidifier, compressed air is first dried by a membrane dryer. The dehumidified stream is then divided over two Bronkhorst $\odot$ flow controllers, with which the flow can be set precisely. One of the air streams is conducted through tubing with semipermeable sides, embedded in a temperature controlled bath, to moisten the air. After leaving the bath, the air stream is conducted through a condenser to remove excess moisture. Both streams are mixed to produce a uniform flow with a relative humidity, the mixing proportions then determine the relative humidity of the air:

$\mathrm{RH}_{\text {set }}=\frac{V_{\text {wet }}}{V_{\text {wet }}+V_{\text {dry }}}$,

where $V_{\text {wet }}$ and $V_{\text {dry }}$ are the wet and dry flow respectively $\left[\mathrm{m}^{3} / \mathrm{s}\right]$.

\section{Results and discussion}

\subsection{Step response}

We have first measured the step response of boards with various thicknesses. In Fig. 5a the deflection in both directions and the calculated length change at half-thickness of the free end of a $4 \mathrm{~mm}$ thick board is shown as a function of time after a step change in the relative humidity from 50 to $90 \%$. The characteristic shape as predicted from the theory is seen. After the step change in relative humidity the board immediately starts to bend up to a maximum deflection, subsequently it more slowly bends back towards its initial $x$-position. The deflective response in the $y$-direction is dependent on the thickness of the board, since it is caused by two processes. Purely geometrically, the bending of an initially straight board causes a change in the y-position of its end. In case of a hygro-expansive material, the length of the board also changes during moisture sorption. This double effect causes the y-position of the end of the board to first decrease before increasing. In a thicker board, which deflects less in the x-direction, expansion dominates and no initial dip in the y-position is recorded.

The deflections in $\mathrm{x}$ - and $\mathrm{y}$-direction are fitted with Eqs. (29) and (31) respectively, as shown in Fig. 5b. The resulting time of maximum deflection $t_{m}$ for the various boards is shown in Fig. 6a as a function of the thickness $d$ of the different boards. A fit with Eq. (24) is added to illustrate the agreement between theory and experiments. The fitted diffusion coefficient has a value of $4.5 \times 10^{-11} \mathrm{~m}^{2} / \mathrm{s}$, which is in accordance with values reported in literature for oak [34-36]. The derived diffusion coefficients for the individual boards are shown in Fig. 6b. The resulting range of $2-6 \times 10^{-11} \mathrm{~m}^{2} / \mathrm{s}$ is relatively narrow for a highly inhomogeneous material such as oak.
Fig. 4 The experimental set-up for measuring the bending of an oak wood board under changing humidity conditions. On the left, the side view of the experimental set-up is shown, the top view is shown on the right

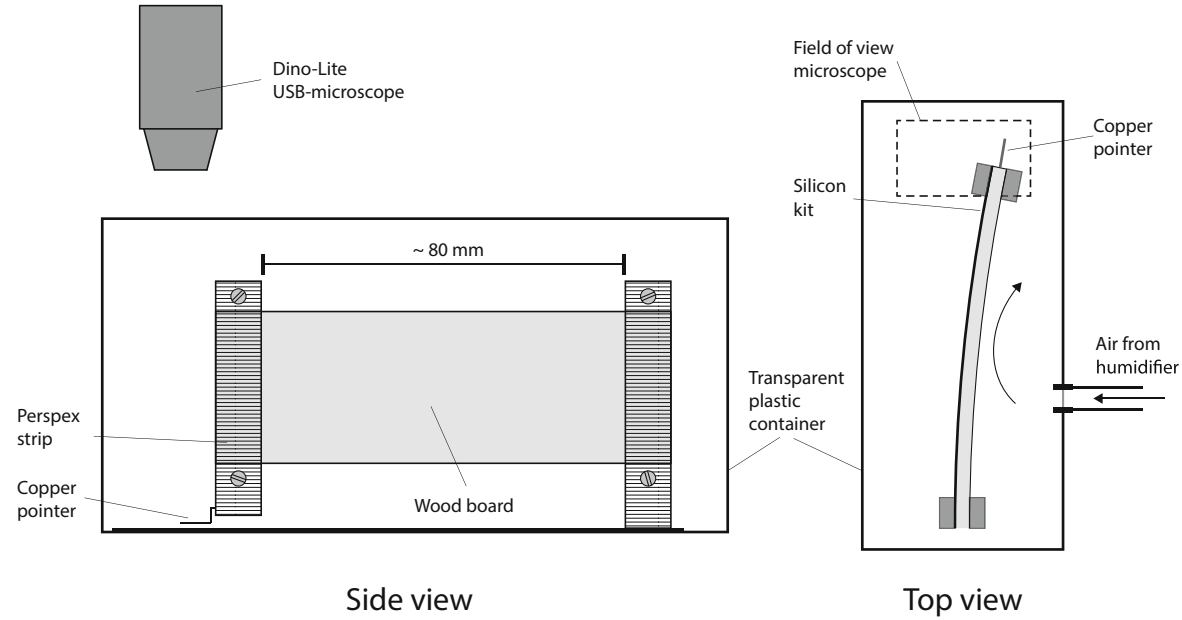


Fig. 5 a The $x$ - and y-deflections $w$ and $v$ of the free end and the length change $\Delta L$ of a clamped, $4 \mathrm{~mm}$ thick board at halfthickness due to a step change in the relative humidity of the ambient air from 50 to $90 \%$, and $\mathbf{b}$ the same experiment fitted with Eqs. (29) and (31)

Fig. 6 a The time of maximum deflection $t_{m}$ as a function of thickness fitted with Eq. (24), and b the derived diffusion coefficients $D$ for the different thicknesses with the average diffusion coefficient resulting from the fit
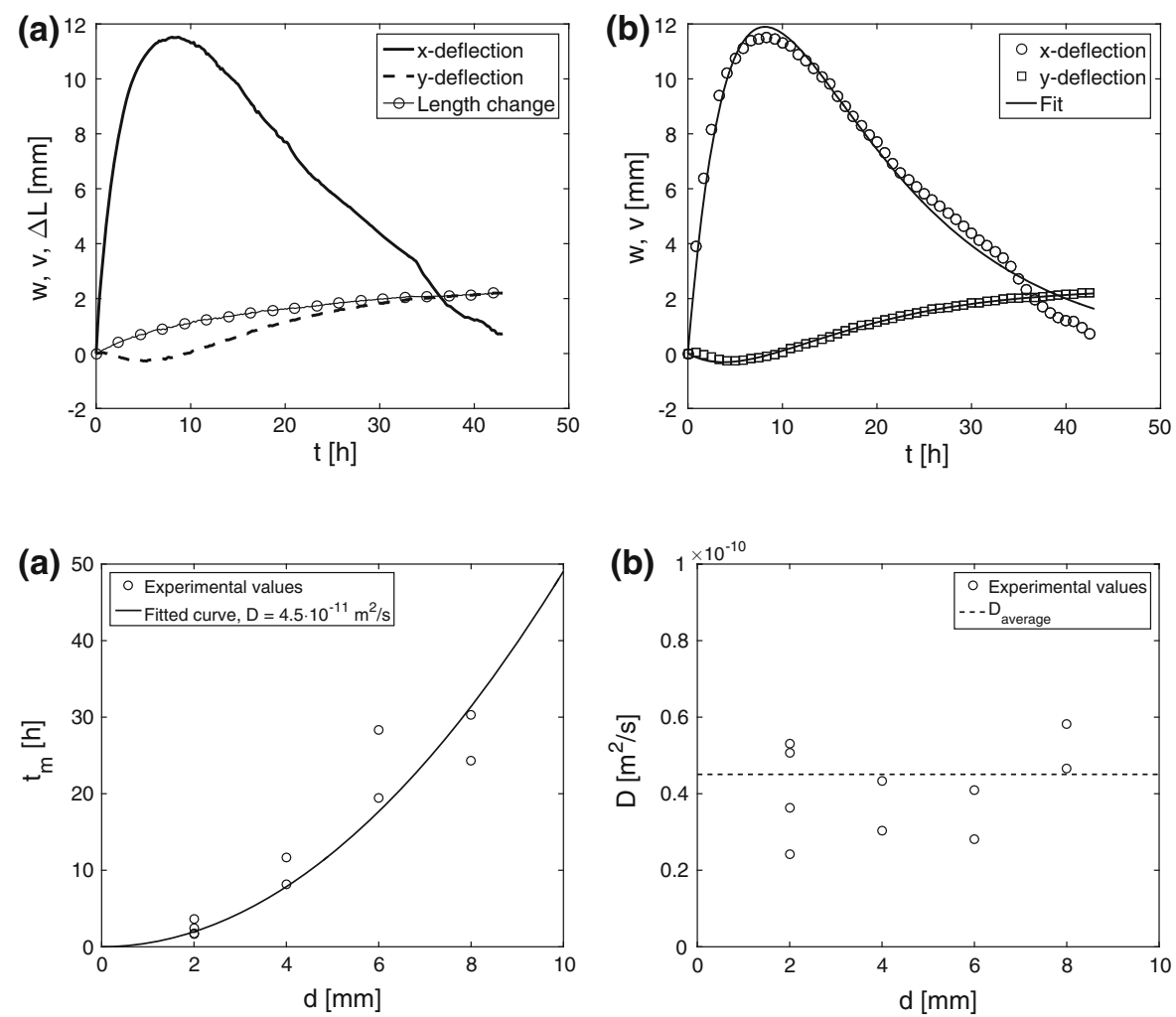

With Eq. (11) the product $\alpha\left(c_{d}-c_{0}\right)$ can be derived from Eq. (31) as $-\left(\beta_{3}+\beta_{4}\right)$. Since the step in ambient relative humidity is known instead of the step in moisture content, an additional operation needs to be performed to obtain the expansion coefficient $\alpha$. The step in moisture content is measured on a sample of the same board using dynamic vapor sorption (DVS). A step in relative humidity from $50 \%$ to $90 \%$ results in $c_{d}-c_{O}=0.11$. This result is used to retrieve the expansion coefficient. The expansion coefficients derived from the asymptote of the expansion after the step change are shown in Fig. 7. The values are between 0.2 and 0.5 , with an average of the derived expansion coefficients (0.35) being in accordance [35] or slightly higher than values reported in literature for the tangential expansion of oak $(\sim 0.3)$ [29]. The scatter in values is most likely caused by differences in initial and final moisture content of the different boards. This can be due to structural inhomogeneities or sorption hysteresis.

With the diffusion and expansion coefficients of the boards determined, the maximum deflection can be scaled according to Eq. (26). The result is shown in Fig. 8, where the theoretical curve $1 / d$ should ideally

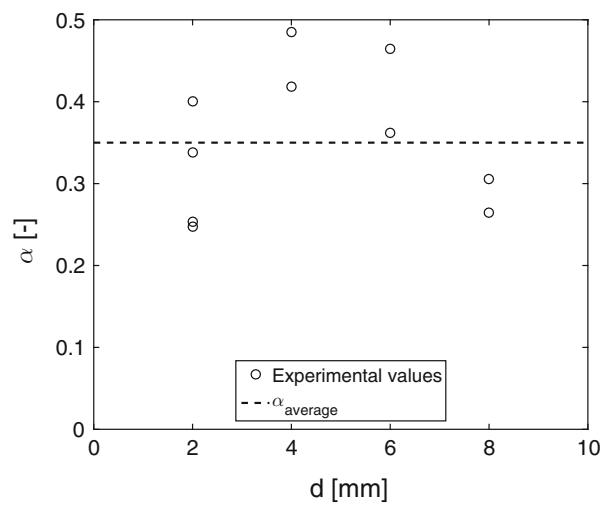

Fig. 7 The linear hygroscopic expansion coefficient $\alpha$ as a function of thickness for different boards as derived from the final expansion of each board

overlay the experimental results. However, a theoretical overestimation of the maximum deflection is seen. Scaling the theoretical line by a factor $1 / 2$ results in a more accurate description of the experimental results. The qualitative scaling behavior of $1 / d$ is thus followed by experiments with different thicknesses, but the absolute values are overestimated, which may have several causes. In the present theory, it is 


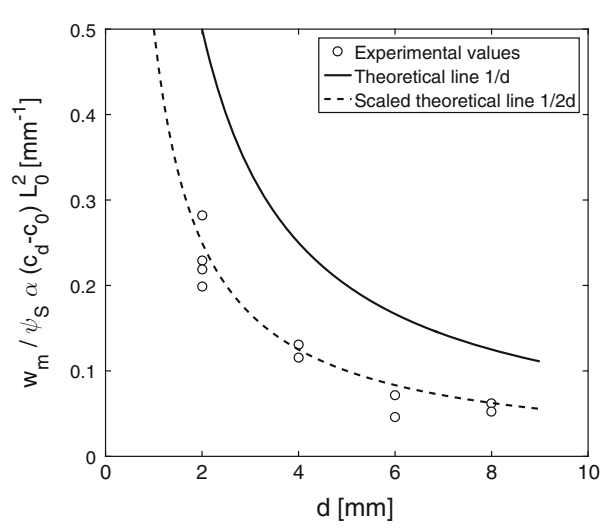

Fig. 8 Scaled values for the maximum deflection $w_{m}$, resulting from application of Eq. (26) to experimental results. The solid curve represents $1 / d$, resulting from the theory, which is also plotted scaled by a factor $1 / 2$ to show the qualitative resemblance of experimental and theoretical values

assumed that the surface of the board reaches equilibrium with the surrounding air immediately. A stationary boundary layer at the surface can, however, increase resistance to moisture sorption. If the transport of moisture is limited by the resistive boundary layer, less asymmetry in the moisture profile is expected over time. As a consequence, the deflective response of the board is less intense. In the experimental set-up used in this study, humid air is blown into the plastic container with a flow such that the air in the container is refreshed at least once a minute. It is therefore presumed that no stagnant boundary layer is formed which attenuates moisture sorption. A more probable cause of the overestimated maximum deflection values is the assumption of wood as a homogeneous, isotropic, and linearly elastic material. Local structural fluctuations can e.g. lower the moisture content asymmetry over time, decreasing the deflection. Also expansion is presumed to follow the change in moisture content immediately, whereas in literature viscoelastic effects are assigned to wood [37]. These time-dependent effects can attenuate the bending of the board. Additional experiments should therefore be performed with a hygro-expansive model-material, which is preferably highly homogeneous and isotropic.

The scaled expansion $\varepsilon^{*}$ as a function of the square root of dimensionless time $t^{*}$ is shown in Fig. 9a for different boards. The theoretically predicted time evolution of $\varepsilon_{t o t} *$ from Fig. 3 is plotted in the same graph. As can be seen, the expansion evolution of the experiments is in reasonable agreement with the predicted course. The experimental slope, however, is higher than the predicted slope. The dimensionless $\mathrm{x}$-deflections $w^{*}$ of the different boards are shown in Fig. 9b. As already observed in Fig. 8, the predicted deflection is approximately twice as large as the scaled experimental results. Similar results were obtained by Holmes et al. [28], which was explained with the use of equilibrium data for scaling kinetic results. Nevertheless, the scaled experiments are all on one main master curve. The general behavior is similar for all experiments, with an initial linear deflection on the square root scale. After the maximum is reached, the boards bend back towards their initial x-position, i.e. towards zero deflection. To compare the experiments to the theory, the theoretical curve for $w_{\text {tot }}^{*}$ scaled by a factor $1 / 2$ is shown too. It is seen that most boards bend back faster towards their initial x-position after the maximum has been reached. The opposite was found in experiments with clay-bearing stone conducted by Scherer and Jimenez Gonzalez [27]; a delay in the post-peak flattening resulted from the dry side being compressed by bending stresses, making it more difficult for water to cause swelling. The stresses which develop due to the bending can have an influence on the moisture transport due to their impact on the chemical potential of water and thus the moisture absorption capacity of the matrix [38]. Since a gradient in chemical potential is the driving force for moisture transport, stresses arising from swelling and shrinkage can have an influence on the moisture distribution over time [39], which again influence the bending.

In the analysis introduced by Scherer and Jimenez Gonzalez [27], the moisture-dependence of the stiffness was taken into account. An estimate of this effect on the bending of the wooden boards is shown in Fig. 10a, in which the E-modulus is varied as $E=10^{b-k c}$, with $b=10$ and $k=1,2,5,10,20$, and 50 (see e.g. Scherer [40] for the influence on the bending). A step in the moisture content $c$ from 0 to 0.2 is considered. As can be seen, the maximum deflection is approximately halved in case of $k=50$. This, however, means that the E-modulus decreases with ten orders of magnitude over the considered moisture content range, which is far from realistic. Saft and Kaliske [35] proposed an E-modulus in the tangential direction which decreases $\sim 50 \%$ over the moisture content range considered here. This would correspond 
Fig. 9 a The scaled expansion $\varepsilon^{*}$ and $\mathbf{b}$ the scaled $\mathrm{x}$-deflection $w^{*}$, plotted as a function of the square root of the normalized time $t^{*}$ for samples with thicknesses of 2, 4, 6 and $8 \mathrm{~mm}$
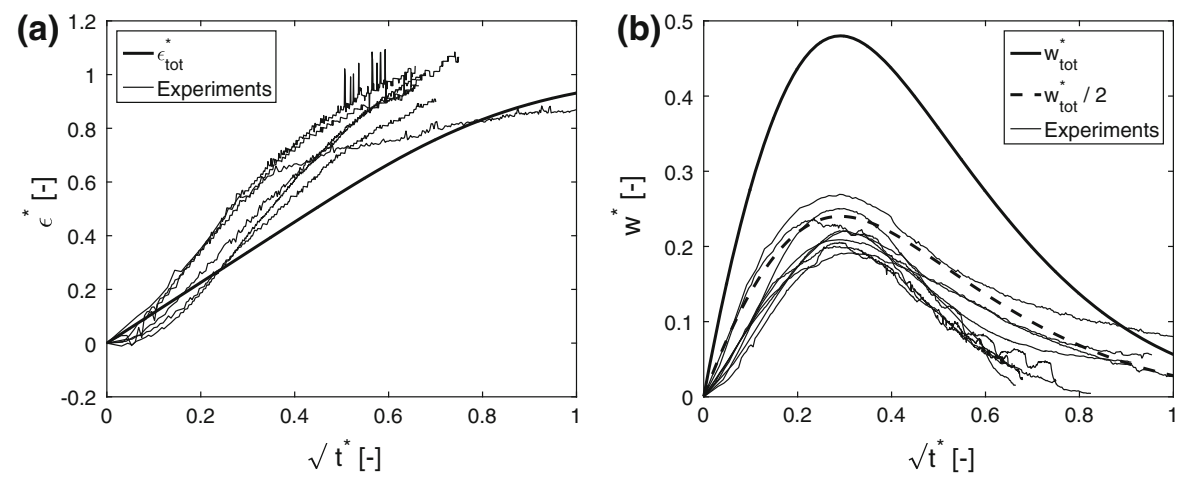

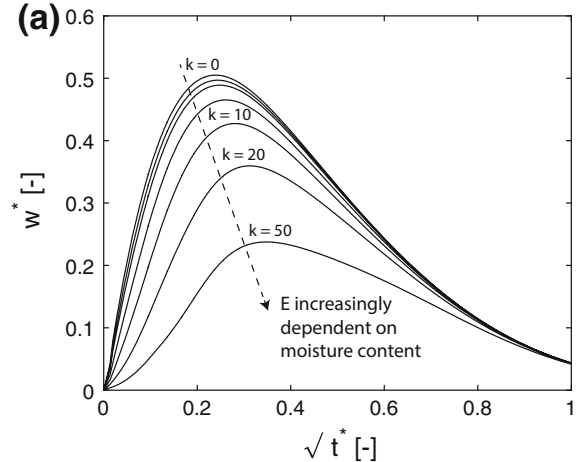

Fig. 10 The scaled deflection $w^{*}$ as a function of $t^{*}$ for a constant diffusion coefficient but different dependencies of the E-modulus on the moisture content $c$ in the form $E=10^{b-k c}$ with $b=10$ and $k=0,1,2,5,10,20$, or 50 , and $\mathbf{b}$ constant E-modulus but different dependencies of the diffusion

to $k \approx 1.5$ in Fig. 10, which has a negligible influence on the deflection of the board over time.

We have so far assumed the diffusion coefficient to be constant. In literature, moisture-dependent values have been measured. The effect on the scaled deflection is shown in Fig. 10b, where a diffusion coefficient of the form $D=D_{0} \mathrm{e}^{k c}$ is assumed. As can be seen, the moisture-dependent diffusion coefficient affects the maximum deflection. A positive $k$, i.e. an increasing diffusion coefficient with increasing moisture content, results in a higher scaled maximum deflection. Conversely, the maximum deflection is attenuated for negative $k$. Also the post-peak flattening is influenced by the moisture-dependent diffusion coefficient. A positive $k$ advances the post-peak flattening, a negative $k$ delays the flattening. A similarly pronounced influence is not found in the experiments presented in Fig. 9b. Furthermore, literature values for the diffusion coefficient generally increase with moisture content $[3,34,41]$, resulting in a higher peak. The

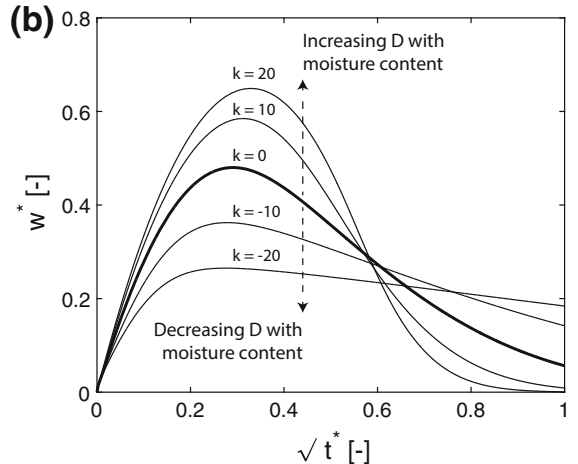

coefficient on the moisture content in the form of $D=D_{o} \mathrm{e}^{k c}$ with $k=-20,-10,0,10,20$. The step in moisture content is assumed to be from 0 to 0.2 . For the moisture-dependent diffusion coefficient an average value over the considered moisture content range is used in $t^{*}$

moisture-dependent diffusion coefficient alone can thus not explain the mismatch between predicted and experimental scaled deflection.

The discrepancy between predicted and experimental scaled deflection may be an indication that transport processes take place other than described by the diffusion equation, as proposed e.g. by Cunningham [42] and Krabbenhoft and Damkilde [43]. Cunningham [42] assumes vapor diffusion takes place in the cell cavities and moisture sorption occurs from the cavities to the cell wall, mathematically described as

$\partial_{t} u=h(l v-m u)$,

$\partial_{t} v=D \partial_{x x} v+h(m u-l v)$,

with $w$ the cell wall moisture content, $v$ the relative humidity in the cell cavities, $h$ an effective mass transfer coefficient, $D$ the diffusion coefficient of water vapor in the cell cavities, and $l$ and $m$ coefficients connecting the vapor and cell wall 
moisture content to a driving potential for moisture sorption. It is assumed here that the ratio $l / m$ is the slope of the sorption curve relating cell wall moisture content to vapor content, and that the water vapor and cell wall moisture are initially and finally in equilibrium. This yields $u_{O}=l / m v_{O}$ and $u_{\infty}=l / m v_{\infty}$, with the subscripts $O$ and $\infty$ indicating initial and final values respectively. Equations (39) and (40) can then be scaled by introducing dimensionless variables:

$v^{*}=\frac{v-v_{0}}{v_{\infty}-v_{0}}, u^{*}=\frac{u-u_{0}}{u_{\infty}-u_{0}}, h^{*}=\frac{l h d^{2}}{D}, m^{*}=\frac{m}{l}$,

$t^{*}=\frac{D t}{d^{2}}, x^{*}=\frac{x}{d}$,

to arrive at

$\partial_{t^{*}} u^{*}=h^{*} m^{*}\left(v^{*}-u^{*}\right)$,

$\partial_{t^{*}} v^{*}=\partial_{x x^{*}} v^{*}+h^{*}\left(u^{*}-v^{*}\right)$.

The effect of sorption from cavities to cell walls on the bending of a board is estimated using Eqs. (42) and (43), complemented with a step change in the vapor content on one surface and a no-flux boundary condition on the other surface. A value for $m^{*}$ of 3.3 is used, which is the reciprocal of a linear sorption curve with a slope of 0.3 . Equations are discretized using a backward Euler scheme and implemented in Matlab. Numerical simulations with different values of $h^{*}$ are performed.

Scaled deflections as a function of the square root of $t^{*}$ are shown in Fig. 11a for different values of $h^{*}$. It can be seen that for slower sorption the maximum deflection is delayed and attenuated compared to the case of immediate equilibrium $\left(h^{*}=\infty\right)$. The delay and attenuation of the maximum scaled deflection are a function of $h^{*}$, as shown in Fig. 11b. Since it can be assumed that $l, h$, and $D$ are no functions of the thickness, $h^{*}$ scales with $d^{2}$. The scaled maximum deflection is then expected to be dependent on board thickness. Experimental results in Fig. 8, however, show that the scaled deflection follows Eq. (26), so that $w_{m}^{*}$ is no function of $d$. Furthermore, the scaled time of maximum deflection would be influenced by board thickness. This would be reflected in a thickness-dependent diffusion coefficient, which is not observed in Fig. 6. A stationary boundary layer at the macroscopic surface attenuating the increase in moisture content at the surface has a similar effect, i.e. a thickness-dependent scaled maximum deflection. It is therefore not likely that moisture sorption to the cell wall or a stationary boundary layer can explain the observed discrepancy between the simple theory and experimental results. Experiments in which the moisture content distribution and bending are measured simultaneously, e.g. using nuclear magnetic resonance, are expected to provide more insight.

\subsection{Frequency response}

To arrive at a rapid means of characterizing its frequency behavior, the deflective step response of a $2 \mathrm{~mm}$ thick board was used according to the procedure introduced in Sect. 2.4. The resulting Bode amplitude plots of the deflection of the board in $\mathrm{x}$ - and $\mathrm{y}$-direction are shown as a function of the frequency in Fig. 12. Three regimes can be distinguished. At low frequencies, the board is seen to deflect to a smaller extent in the $\mathrm{x}$-direction, but its amplitude in the y-direction reaches a constant value. During these slow fluctuations, the asymmetry in the moisture content distribution is minor, resulting in small $\mathrm{x}$-deflections. The moisture penetration is high, resulting in a high amplitude in the y-deflection. In an intermediate frequency interval, the board reacts heavily to the imposed fluctuations in relative humidity. For increasing frequencies, the amplitude in both $\mathrm{x}$ - and $\mathrm{y}$-deflection is seen to decline; the variations in relative humidity are too fast to follow, i.e. the moisture does not penetrate far enough into the material to cause deflections.

In order to verify the frequency response predicted from its step response, we have conducted experiments with the same board where the relative humidity was varied sinusoidally at different frequencies. The equilibrium relative humidity was $50 \%$ and the amplitude $40 \%$ in all experiments. The result of one of these measurements for a board with a thickness of $2 \mathrm{~mm}$ is given in Fig. 13, and shown in the animation (Online Resource 1). The board is seen to deflect cyclically to the imposed relative humidity variations. Furthermore it is seen that the deflection during moisture absorption is slightly larger than during desorption. This can be explained with the sorption curve of wood, which has an S-shape with a relatively large increase at high relative humidity [3]. Although the input, i.e. the relative humidity, has a pure sinusoidal shape, the non-linearity of the sorption 


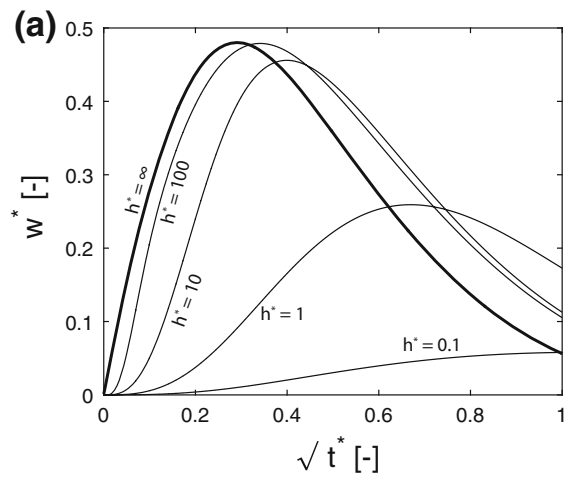

Fig. 11 a The scaled deflection $w^{*}$ as a function of the square root of $t^{*}$ for vapor transport in the cell cavities of the wood and moisture sorption to the cell wall for different values of $h^{*}$. The thick solid line represents Eq. (15), i.e. instantaneous

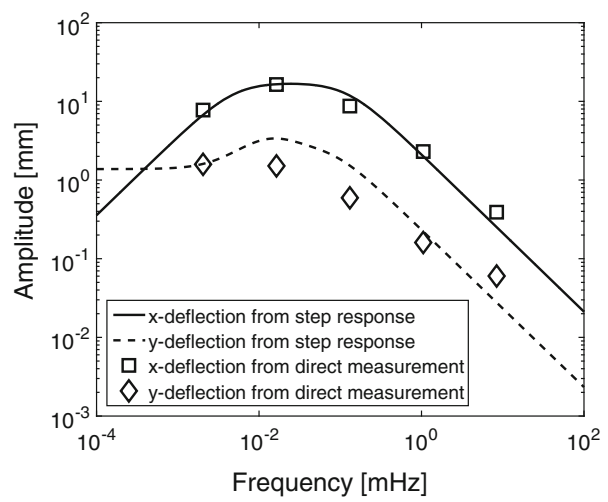

Fig. 12 Bode amplitude plots for the $\mathrm{x}$ - and $\mathrm{y}$-deflection of the free end of a $2 \mathrm{~mm}$ thick board, which results from application of Eqs. (36) and (37) to a step change from a relative humidity of $50-90 \%$. For the experimental values, the slowest variation is $134 \mathrm{~h}$, the fastest $2 \mathrm{~min}$

curve causes the moisture content at the exposed surface to follow a transformed course over time. In Fig. 12 we have plotted the amplitudes from individual experiments at different frequencies (periods of $134 \mathrm{~h}$ to $2 \mathrm{~min}$ ). As can be seen these results overlap well with the frequency response as calculated from a single step experiment. Some quantitative deviations between predicted and experimentally found values are encountered, especially with the y-deflection. As mentioned earlier, the y-deflection has a dual source: it arises as a geometrical consequence of the bending and is caused by expansion/shrinkage. This explains the peak in the predicted curve, which is not seen to be confirmed by experiments. Nevertheless, the agreement between prediction based on the assumption of

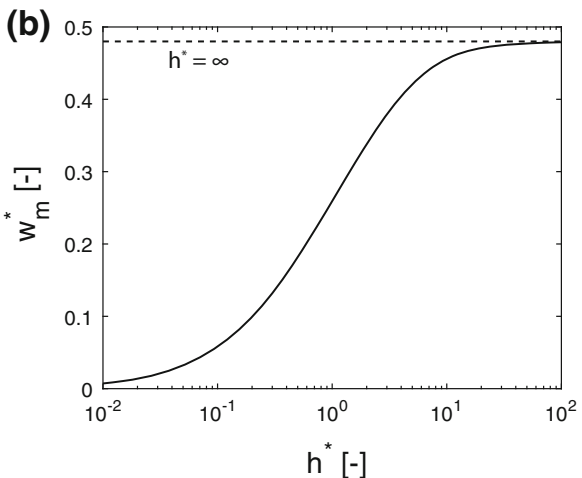

equilibrium between water vapor and moisture in the cell wall. b The scaled maximum deflection as a function of $h^{*}$, for $m^{*}=3.3$. The dotted line indicates the maximum deflection of Eq. (15)

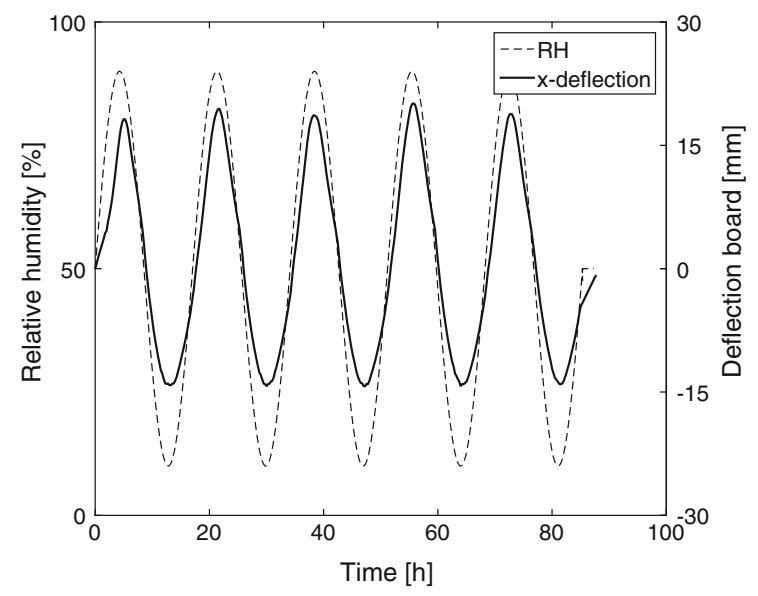

Fig. 13 Experimental $\mathrm{x}$-deflection of a $2 \mathrm{~mm}$ thick board during sinusoidal relative humidity $(\mathrm{RH})$ fluctuations with a period of $17 \mathrm{~h}$

linear system behavior and actual experiments is striking. Despite non-linear effects affecting moisture transport and expansion in wood which are often found in literature, the behavior in these experiments is seen to be fairly linear. This result enables simple and insightful prediction of hygromorphic behavior of wood. Instead of measuring the response at various frequencies, which is time-consuming, the frequency behavior can be predicted with a short experiment.

\section{Conclusions}

A simple theory is proposed based on the diffusion equation and linear elasticity to analyze experiments 
with oak boards exposed unilaterally to moisture. The diffusion coefficient and linear hygroscopic expansion coefficient of wood can be derived accordingly, which are in agreement with literature values. The scaling law concerning the maximum deflection of the board during a step change in the relative humidity is seen to describe the experiments well qualitatively, but overestimates the deflection quantitatively. Possible causes are the oversimplification of the involved processes, neglecting non-linear, non-elastic effects, local inhomogeneities and the influence of stresses on moisture transport. From a simple parameter study we can conclude that moisture-dependent values for the E-modulus and the diffusion coefficient cannot explain the mismatch in scaled deflection between the simple theory and experimental results. A different moisture transport model, with vapor diffusion and sorption to the cell wall, cannot explain the observed discrepancy either due to a thickness-dependence of the scaled deflection, which is not seen in experiments.

Approximation of the bending behavior of the wood board as linear system behavior allows the derivation of the frequency response from its step response. The behavior is well captured, reflected in the agreement in shape of the Bode plots predicted from a step response and direct measurement with sinusoidal relative humidity fluctuations. The ability to predict the frequency behavior of the board from a relatively short experiment is an encouraging result, especially in combination with the scalable responses for different boards. Step responses of thick boards and frequency behavior at slow fluctuations, both highly time-consuming experiments, can thus be predicted with a short experiment with a thin board. This allows a fast characterization of the hygromorphic response of a material.

A promising extension of the experimental part of the study should be sought in the simultaneous measurement of the deflection and the local moisture content. The deflection can be assessed optically, as shown in this study, whereas the moisture content could be measured non-destructively by nuclear magnetic resonance. In this way the moisture content profile can be related directly to the hygromorphic response at any time. This combination provides more information about the moisture transport during the bending experiment, and possibly elucidates the interrelation between the stress distribution and moisture transport.
Acknowledgements The authors would like to thank J. H. J. Dalderop for his technical support, A. H. Arends for his help with the bending theory, and G. Fransen for the preparation of samples. We thank G. W. Scherer, as well as H. L. Schellen, R. A. Luimes, and A. S. J. Suiker for fruitful discussions. This work is part of the research program Science4Arts, financed by the Netherlands Organization for Scientific Research (NWO), and carried out in the Darcy Center for porous media research.

\section{Compliance with ethical standards}

Conflict of interest The authors declare that they have no conflict of interest.

Open Access This article is distributed under the terms of the Creative Commons Attribution 4.0 International License (http:// creativecommons.org/licenses/by/4.0/), which permits unrestricted use, distribution, and reproduction in any medium, provided you give appropriate credit to the original author(s) and the source, provide a link to the Creative Commons license, and indicate if changes were made.

\section{References}

1. Reyssat E, Mahadevan L (2009) Hygromorphs: from pine cones to biomimetic bilayers. J R Soc Interface 6:951-957

2. Siau JF (1984) Transport processes in wood. SpringerVerlag, Berlin

3. Skaar C (1988) Wood-water relations. Springer-Verlag, Berlin

4. Larsen KE, Marstein N (2000) Conservation of historic timber structures: an ecological approach. ButterworthHeinemann, Oxford

5. Reichert S, Menges A, Correa D (2015) Meteorosensitive architecture: biomimetic building skins based on materially embedded and hygroscopically enabled responsiveness. Comput Aided Des 60:50-69

6. Holstov A, Bridgens B, Farmer G (2015) Hygromorphic materials for sustainable responsive architecture. Constr Build Mater 98:570-582

7. Brandao A, Perré P (1996) The "flying wood"—a quick test to characterise the drying behavior of tropical wood. In: Fifth international IUFRO wood drying conference, pp 315-324

8. Allegretti O, Rémond R, Perré P (2003) A new experimental device for non-symmetrical drying tests - experimental and numerical results for free and constrained samples. In: Ispas M, Campean M, Cismaru I, Marinescu I, Budau G (eds) Proceedings 8th international IUFRO wood drying conference, pp 65-70

9. Allegretti O, Ferrari S (2007) Characterization of the drying behaviour of some temperate and tropical hardwoods. In: Proceedings of the 1 st international scientific conference on hardwood processing, pp 285-290

10. Uetimane Junior E, Allegretti O, Terziev N, Söderström O (2010) Application of non-symmetrical drying tests for assessment of drying behaviour of ntholo (Pseudolachnostylis maprounaefolie PAX). Holzforschung 64:363-368 
11. Rémond R, De La Cruz M, Aléon D, Perré P (2013) Investigation of oscillating climates for wood drying using the flying wood test and loaded beams: need for a new mechano-sorptive model. Maderas Ciencia y Technolo 15:269-280

12. Dionisi Vici P, Mazzanti P, Uzielli L (2006) Mechanical response of wooden boards subjected to humidity step variations: climatic chamber measurements and fitted mathematical models. J Cult Herit 7:37-48

13. Engelund ET, Thygesen LG, Svensson S, Hill CAS (2013) A critical discussion of the physics of wood-water interactions. Wood Sci Technol 47:141-161

14. van Duin P (2011) Panels in furniture: observations and conservation issues. In: Phenix A, Chui SA (eds) Facing the challenges of panel paintings conservation: trends, treatments and training. The Getty Conservation Institute, Los Angeles, pp 92-103

15. Blumer S, Serrano E, Gustafsson PJ, Niemz P (2011) Moisture induced stresses and deformations in parquet floors. In: Bucur V (ed) Delamination in wood, wood products and wood-based composites. Springer, Dordrecht, pp 365-378

16. Schellen HL (2002) Heating monumental churches: indoor climate and preservation of cultural heritage. Dissertation, Eindhoven University of Technology

17. Chomcharn A, Skaar C (1983) Dynamic sorption and hygroexpansion of wood wafers exposed to sinusoidally varying humidity. Wood Sci Technol 17:259-277

18. Derome D, Rafsanjani A, Patera A, Guyer R, Carmeliet J (2012) Hygromorphic behaviour of cellular material: hysteretic swelling and shrinkage of wood probed by phase contrast X-ray tomography. Philos Mag 92:1-21

19. Gauvin C, Jullien D, Dupré J-C, Gril J (2014) Image correlation to evaluate the influence of hygrothermal loading on wood. Strain 50:428-435

20. Lanvermann C, Wittel FK, Niemz P (2014) Full-field moisture induced deformation in Norway spruce: intra-ring variation of transverse swelling. Eur J Wood Wood Prod 72:43-52

21. Senni L, Caponero M, Casieri C, Felli F, De Luca F (2010) Moisture content and strain relation in wood by Bragg grating sensor and unilateral NMR. Wood Sci Technol 44:165-175

22. Caré S, Bornert M, Bertrand F, Lenoir N (2012) Local moisture content and swelling strain in wood investigated by NMR and X-ray microtomography. In: Proceeding of the 15th international conference on experimental mechanics

23. Scherer GW (1992) Bending of gel beams: method for characterizing elastic properties and permeability. J NonCryst Solids 142:18-35

24. Vichit-Vadakan W, Scherer GW (2003) Measuring permeability and stress relaxation of young cement paste by beam bending. Cem Concr Res 33:1925-1932

25. Scherer GW, Prévost JH, Wang Z-H (2009) Bending of a poroelastic beam with lateral diffusion. Int J Solids Struct 46:3451-3462
26. Zhang J, Scherer GW (2012) Permeability of shale by the beam-bending method. Int $\mathrm{J}$ Rock Mech Min Sci 53:179-191

27. Scherer GW, Jimenez Gonzalez I (2005) Characterization of swelling in clay-bearing stone. In: Stone decay in the architectural environment: Geological Society of America Special Paper 390, pp 51-61

28. Holmes DP, Roché M, Sinha T, Stone HA (2011) Bending and twisting of soft materials by non-homogeneous swelling. Soft Matter 7:5188-5193

29. Forest Products Laboratory (2010) Wood handbook-wood as an engineering material. United States Department of Agriculture, Madison

30. Yang T, Ma E (2015) Dynamic sorption and hygroexpansion of wood subjected to cyclic relative humidity changes. II. The effect of temperature. BioResources 10:1675-1685

31. Strauss WA (2008) Partial differential equations: an introduction, 2nd edn. John Wiley and Sons, Hoboken

32. Boley BA, Weiner JH (1997) Theory of thermal stresses. Dover, Mineola

33. Franklin GF, Powell JD, Emami-Naeini A (1994) Feedback control of dynamic systems, 3rd edn. Addison-Wesley Publishing, Reading

34. Simpson WT (1993) Determination and use of moisture diffusion coefficient to characterize drying of northern red oak (Quercus rubra). Wood Sci Technol 27:409-420

35. Saft S, Kaliske M (2013) A hybrid interface-element for the simulation of moisture induced cracks in wood. Eng Fract Mech 102:32-50

36. Gezici-Koç Ö, Erich SJF, Huinink HP, van der Ven LGJ, Adan OCG (2017) Bound and free water distribution in wood during water uptake and drying as measured by $1 \mathrm{D}$ magnetic resonance imaging. Cellulose 24:535-553

37. Schniewind AP, Barrett JD (1972) Wood as a linear orthotropic viscoelastic material. Wood Sci Technol 6:43-57

38. Derrien K, Gilormini P (2007) The effect of applied stresses on the equilibrium moisture content in polymers. Scr Mater 56:297-299

39. Sar BE, Fréour S, Davies P, Jacquemin F (2012) Coupling moisture diffusion and internal mechanical states in polymers - a thermodynamical approach. Eur J Mech A Solids 36:38-43

40. Scherer GW (1987) Drying gels: III. Warping plate. J NonCryst Solids 91:83-100

41. Baronas R, Ivanauskas F, Juodeikiene I, Kajalavicius A (2001) Modelling of moisture movement in wood during outdoor storage. Nonlinear Anal Model Control 6:3-14

42. Cunningham MJ (1995) A model to explain "anomalous" moisture sorption in wood under step function driving forces. Wood Fiber Sci 27:265-277

43. Krabbenhoft K, Damkilde L (2004) A model for nonFickian moisture transfer in wood. Mater Struct 37:615-622 